\title{
A new impact dynamics model of a clearance joint considering the adhesive effects in space environment
}

\author{
Ruiting Tong ${ }^{1, *}$, Zefen Quan $^{2}$, Qi Wan ${ }^{1}$, Xiaojun Fu ${ }^{1}$, and Geng Liu ${ }^{1}$ \\ ${ }^{1}$ Shaanxi Engineering Laboratory for Transmissions and Controls, Northwestern Polytechnical University, 710072 Xi'an, P. R. China \\ ${ }^{2}$ Shanghai Aircraft Design and Research Institute, 200436 Shanghai, P. R. China
}

\begin{abstract}
In space environment, there is severe adhesive effect, and the impact contact process shows different phenomenon due to the adhesive forces. In this paper, a new impact dynamics model of a clearance joint is developed considering the adhesive effects. The Hertz contact model, L-J potential and Dugdale model are combined, and an adhesive force term is included in the original hybrid contact force model. Taking a clearance joint as an example, the theory of adhesive forces during the impact contact process is given, and the influence of the adhesive forces is investigated. Negative forces are discovered at the beginning of the contact, and the contact forces of the modified model are higher than the hybrid model. Besides, the indentation depth is also higher than the hybrid model. The energy conservation principle is employed to explain the phenomena, and the contact forces will be underestimated if the adhesive effects are ignored. This work could be contributed to estimating the contact forces and friction forces more reasonablely in the space environment.
\end{abstract}

\section{Introduction}

In space environment, the microgravity and high vacuum are the typical characteristics. Under the microgravity, there are irregular impacts all the time, and the high vacuum induces severe adhesive effects due to clean contact surfaces. To realize movement or power transmission, the motion of the mechanical mechanism is necessary, and the clearance is evitable. For a clearance joint, even a small clearance can produce an obvious influence on the dynamic characteristics, wear and lifetime of mechanical systems [1-2], and the impact will greatly influence the friction properties [3].

Many works are done on modelling the impact contact problems, and the clearance joints are always taken as examples. Two classical models, Hertz model and Persson model [4-5] are widely used to investigate the contact behaviors of the clearance joint. Considering the energy loss, Lankarani and Nikravesh [6] improved the Hertz contact model by incorporating a hysteresis damping function, and a Lankarani-Nikravesh(L-N) model was developed. For a cylindrical clearance joint, Liu et al. [7-8] developed an approximate model, and it was more precise than the Hertz model and Persson model. A hybrid contact force model was developed by Bai and Zhao [9], in which the L-N model and an improved elastic foundation model were combined, and the model showed a wide applicable scope. Ma et al. [10] also presented a hybrid contact force model by extending the L-N contact force model and the elastic foundation model, and the hybrid model was validated by comparing with experimental results. Alves et al. [11] compared various contact force models, and pointed out that the dynamic behaviors of contact bodies greatly depended on the contact force model. A nonlinear model for a clearance joint was introduced by Wang et al. [12], and the impact velocities, material properties and geometry dimensions of the journal and bearing were considered, which made it get a wide suitable range.

The adhesive effects are not considered in the references above due to that they are so slight in ambient environment and could be neglected. In the space environment, adhesion forces are more important between clean metal surfaces than the ambient environment, and the friction coefficient in vacuum environment could be six times higher than the case of ambient environment [13]. Therefore, the adhesive effects should be considered in the impact dynamics model in space environment.

Several classical theories could consider the influence of the adhesion force in contact force models. Bradley [14] investigated the adhesive contact between two contact rigid spheres, and the expression of the adhesion was given. Derjaguin-Muller-Toporov (DMT) theory [15] was developed based on the adhesive contact model between a ball and a plane, and a relationship between the adhesion force and the radius of the ball was described. The Johnson-Kendall-Roberts (JKR) theory of Johnson et al. [16] presented the formula of the adhesive contact force between two elastic spherical solid surfaces. Tabor [17] defined a Tabor parameter, and the competition between the DMT theory and JKR theory could be solved. By using the Dugdale model, Maugis [18] developed a Maugis-Dugdale (M-D) theory that was

Corresponding author:tongruiting@nwpu.edu.cn 
a JKR-DMT transition, and the intermediate region between the JKR theory and DMT theory was discovered. Extending the M-D theory, Baney and Hui [19] developed a cohesive zone model to describe the adhesion between long cylinders in contact. Based on these theories, more research in this area were carried out, while the adhesion theories above were not used in impact contact force models.

In this paper, taking a clearance joint as an example, based on the hybrid contact force model [9], a modified contact force model is developed combining the adhesive effects in space environment. The theory of adhesive forces during the impact process is given, and an example is presented in the final. The results of these two models are compared, and the adhesive effects are discussed.

\section{Model description}

\subsection{Hybrid contact force model [9]}

Bai and Zhao [9] developed a hybrid contact force model, and the contact force is

$$
F_{\text {Nhyb }}=K_{n} \delta^{n}+D_{m o d} \dot{\delta}
$$

where $K_{n}$ is a nonlinear stiffness coefficient, $\delta$ is the deformation, the exponent $n$ is equal to $1.5, D_{\text {mod }}$ is the modified damping coefficient and it is related to the nonlinear stiffness coefficient, $K_{n}$, and $\dot{\delta}$ is the relative deformation velocity. Based on the Persson contact model, the relationship between the normal force $P$ and the deformation $\delta$ is

$$
P=\frac{1}{2} \pi \delta E^{*} \sqrt{\frac{\delta}{2(\Delta R+\delta)}}
$$

where $E^{*}$ is the equivalent elastic modulus, and $\Delta R$ is difference between the radii of the bearing and journal.

$$
\begin{gathered}
\frac{1}{E^{*}}=\frac{1-\mu_{B}^{2}}{E_{B}}+\frac{1-\mu_{J}^{2}}{E_{J}} \\
\Delta R=R_{B}-R_{J}
\end{gathered}
$$

where $E_{B}$ and $E_{J}$ are the elastic modulus, $\mu_{B}$ and $\mu_{J}$ are the Poisson's ratio, and $R_{B}$ and $R_{J}$ are the radii of the bearing and journal, respectively.

The nonlinear stiffness coefficient can be obtained as

$$
\begin{aligned}
K_{n}= & \frac{d P}{d \delta}=\frac{d\left(\frac{1}{2} \pi \delta E^{*} \sqrt{\frac{\delta}{2(\Delta R+\delta)}}\right)}{d \delta} \\
= & \frac{1}{8} \pi E^{*} \sqrt{\frac{2 \delta\left[3\left(R_{B}-R_{J}\right)+2 \delta\right]^{2}}{\left(R_{B}-R_{J}+\delta\right)^{3}}}
\end{aligned}
$$

The modified damping coefficient can be obtained as

$$
D_{\text {mod }}=\frac{3 K_{n}\left(1-c_{e}^{2}\right) e^{2\left(1-c_{e}\right)} \delta^{n}}{4 \dot{\delta}_{0}}
$$

where $c_{e}$ is the coefficient of restitution, and $\dot{\delta}_{0}$ is the initial impact velocity, which are used to represent impact.

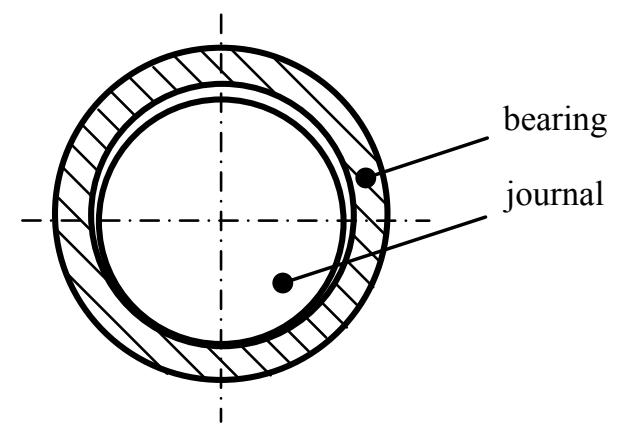

Fig. 1. A simplified clearance joint

\subsection{Adhesive force model}

If the adhesive effects are considered, there will be adhesive pressure $p_{A}$ on the interface in addition to the Hertzian pressure $p_{H}$. The Hertzian pressure acts on the region $|r|<a$, and the adhesive pressure acts on the region $|r|<c$, as shown in Fig. 2 .

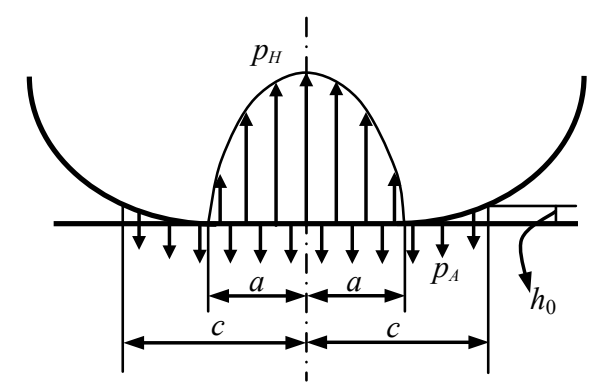

Fig. 2. Adhesion at the interface of the contact bodies [20]

Generally, the adhesive pressure $p_{A}$ is assumed as a function of separation $z$ between the two contact surfaces, and it follows the law derived from the Lennard-Jones (L-J) potential

$$
p_{A}(z)=\frac{8 \omega}{3 z_{0}}\left[\left(\frac{z}{z_{0}}\right)^{-3}-\left(\frac{z}{z_{0}}\right)^{-9}\right]
$$

where $\omega$ is the adhesion energy, and $z_{0}$ is the equilibrium separation of the surfaces.

For a two-dimensional adhesive contact between a cylinder and a plane, Baney and Hui [19] simplified the L-J force, as shown in Fig. 3. The adhesive force out of the contact area was assumed as a constant $\sigma_{0} \bullet A\left(\sigma_{0}\right.$ is a constant called theoretical stress, and $\sigma_{0}=\omega / h_{0}$, $h_{0}=0.97 z_{0}, A$ is the contact area of the region $\left.a \leq|r| \leq c\right)$, up to a maximum separation $h_{0}$, and it falls to zero if the separation is greater than $h_{0}$. 


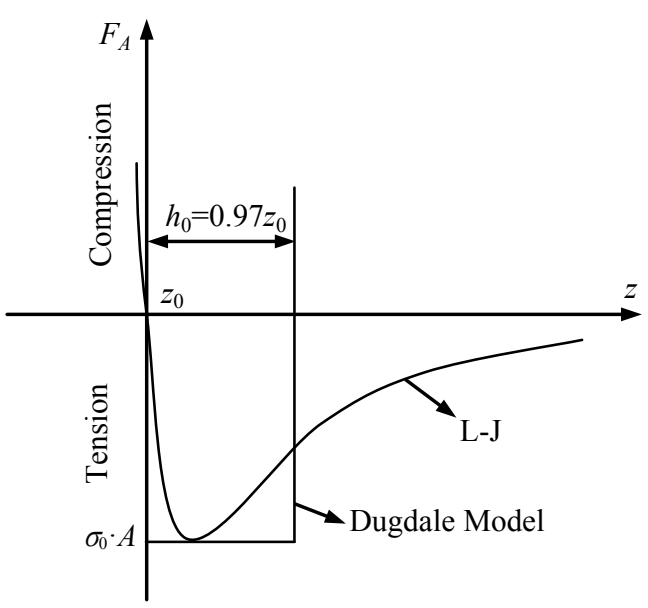

Fig. 3. Adhesive forces derived from the L-J potential and the Dugdale model in fracture mechanics

The pressure caused by the adhesive force for cylinders can be expressed as

$$
p_{A}(r)=\left\{\begin{array}{cr}
\sigma_{0} & a \leq|r| \leq c \\
\frac{\sigma_{0}}{\pi} \tan ^{-1} \sqrt{\frac{c^{2}-a^{2}}{a^{2}-r^{2}}} & |r|<a
\end{array}\right.
$$

When $|r|<a$, the compression plays the dominant role, so the pressure caused by the adhesive force in the range of $|r|<a$ is ignored in this paper.

Considering the clearance joint in Fig. 1, when the impact occurs, the deformation is shown in Fig. 4. We define that $\overparen{D A}=a$ is the Hertzian contact half-width, and $\overparen{A C}=c-a$ is the region where adhesive forces are considered. Then the adhesive region can be rewritten as $\overparen{A C}=R_{B} \bullet \theta$.

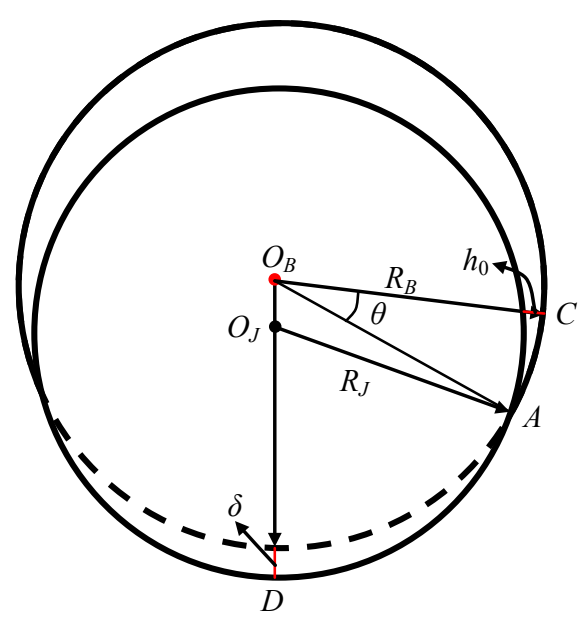

Fig. 4. Deformation in impact contact between the journal and bearing

Based on the definition and the simplication, we can obtain the adhesive pressure

$$
p_{A}=\sigma_{0} \quad a \leq|r| \leq c
$$

and the adhesive force in a two-dimensional problem can be written as

$$
F_{A}=p_{A} \cdot \overparen{A C}=\sigma_{0} \cdot \overparen{A C}=\sigma_{0} \cdot R_{B} \bullet \theta \quad a \leq|r| \leq c
$$

\subsection{Modified contact force model}

Coupling the hybrid contact force model [9] and the adhesive force model in this paper, we can obtain a modified contact force model as follows

$$
F_{N \text { mod }}=K_{n} \delta^{n}+D_{\text {mod }} \dot{\delta}-F_{A}
$$

In equation (11), the first two terms are the hybrid contact force, and the parameters are the same as Bai and Zhao's model [9].

\section{Example}

Taking a clearance joint as an example, the materials of the journal and bearing are the same, and the parameters used in the simulation are listed in Table 1. Using the software Matlab, both the hybrid contact force model and modified contact force model are simulated, and the results are compared in Fig. 5-Fig. 7.

Table 1. Parameters of the example.

\begin{tabular}{|c|c|}
\hline Parameters & Value \\
\hline Material & Iron $(\mathrm{BCC} \mathrm{Fe})$ \\
\hline Elastic modulus $E / \mathrm{Pa}$ & $2.11 \times 10^{11}$ \\
\hline Poisson's ratio $\mu$ & 0.3 \\
\hline Radius of the journal $R_{J} / \mathrm{m}$ & $9.5 \times 10^{-3}$ \\
\hline Radius of the bearing $R_{B} / \mathrm{m}$ & $10.0 \times 10^{-3}$ \\
\hline Adhesion energy $\omega / \mathrm{J} \cdot \mathrm{m}^{-2}$ & $3.07[21]$ \\
\hline Equilibrium separation $z_{0} / \mathrm{m}$ & $1.58 \times 10^{-10}$ \\
\hline Mass of the journal $m / \mathrm{kg}$ & 0.08 \\
\hline Initial impact velocity $\dot{\delta}_{0} / \mathrm{m}^{-1} \mathrm{~s}^{-1}$ & 0.3 \\
\hline Coefficient of restitution $c_{\mathrm{e}}$ & 0.8 \\
\hline
\end{tabular}

Fig. 5 shows the relationship between the time and the contact forces. The negative contact forces at the beginning of the impact contact indicates that there are obvious adhesive effects. When the journal just contacts the bearing, the adhesive forces act on the journal, which means that the journal is accelerated, and the contact forces of the modified model are higher than the hybrid model. In addition, due to that the energy done by the adhesive forces acts on the journal, so the impact contact time is longer for the modified model compared with the 
hybrid model. At the end of the impact process, the contact forces also show negative values to show the adhesive effects.

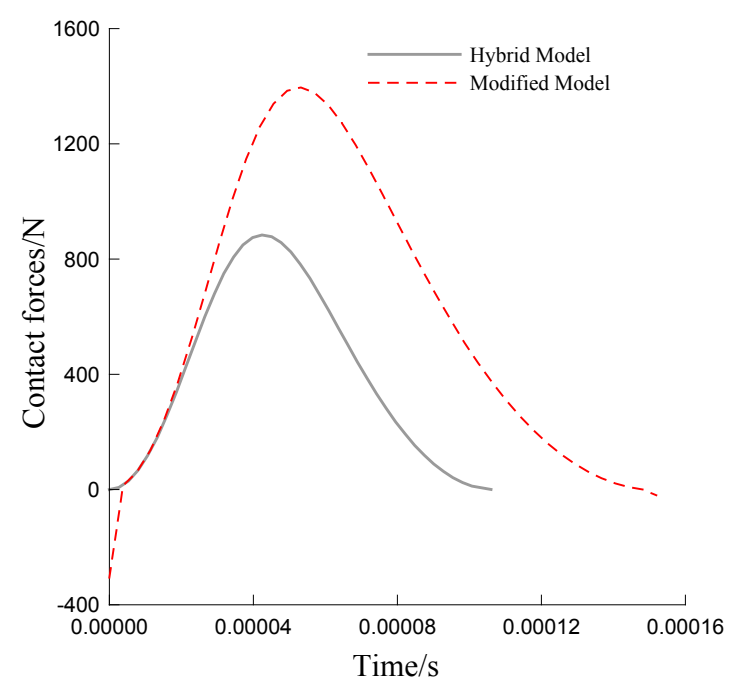

Fig. 5. The relationship between the time and the contact forces

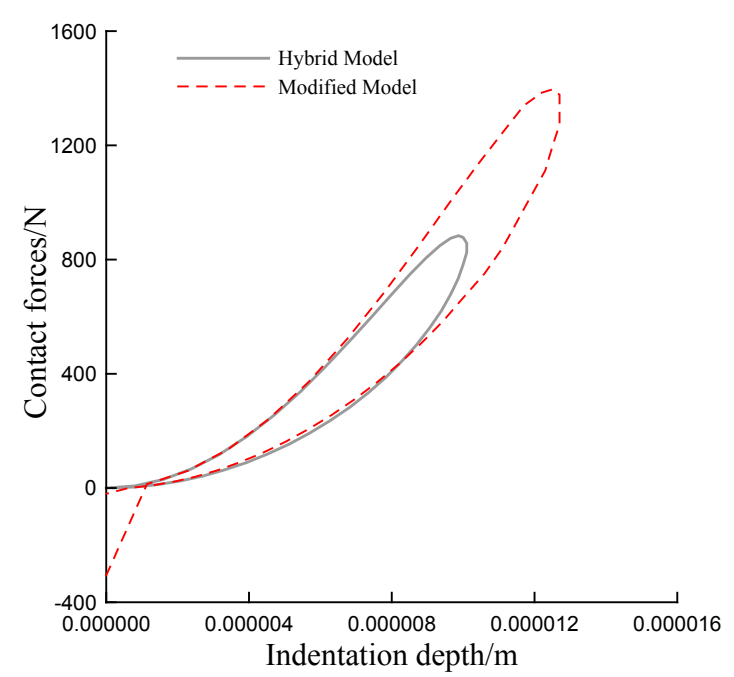

Fig. 6. The relationship between the indentation depth and the contact forces

The impact-restitution curves are given in Fig. 6, and the indentation depth and contact forces obtained from the modified model are higher than the hybrid model. From the view of energy conservation principle, the kinetic energy of the journal will be converted to the mechanical energy during the impact process. In the modified model, the total energy includes the work done by the adhesive forces, so the indentation depth and the contact forces are higher than the hybrid model. Furthermore, the energy conversion process should be the reason for the phenomenon in Fig. 7.

\section{Conclusions}

Considering the adhesive effects in the space environment, a modified contact force model is developed based on the Hertz contact model, L-J potential and Dugdale model. A clearance joint is used as an example, and the relationship between the time and contact forces, the indentation depth and the contact forces and the time and indentation depth are investigated. Some conclusions are drawn as follows:

(1) If the adhesive effects are considered, there will be negative contact forces, and the contact forces of the modified model are higher than the hybrid model.

(2) The kinetic energy and the work done by the adhesive forces are converted to the mechanical energy in the impact process, so the indentation depth of the modified model is higher than the hybrid model.

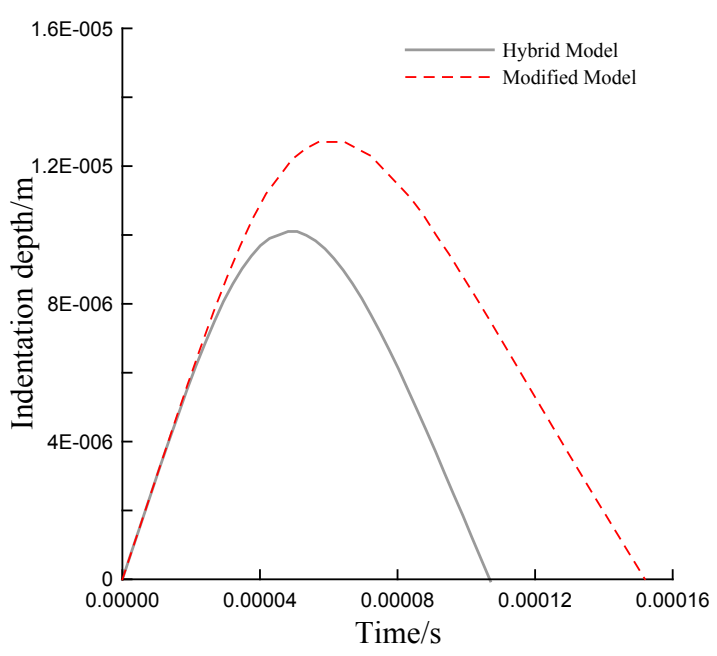

Fig. 7. The relationship between the time and the indentation depth

The authors would like to thank the National Natural Science Foundation of China (51675429), Key Project of National Natural Science Foundation of China (51535009), the Fundamental Research Funds for the Central Universities (31020190503004) and the 111 Project (B13044) for their financial support.

\section{References}

1. S. Erkaya, J. Mech. Sci. Technol. 26, 1419-1430 (2012)

2. X.P. Wang, G. Liu, J. Mech. Sci. Technol. 29, 41134120 (2015)

3. R.T. Tong, G. Liu, Microgravity Sci. Tec. 31, 85-94 (2019)

4. M. Ciavarella, P. Decuzzi, Int. J. Solids Struct. 38, 4507-4523 (2001)

5. M. Ciavarella, P. Decuzzi, Int. J. Solids Struct. 38, 4523-4533 (2001)

6. H.M. Lankarani, P.E. Nikravesh, J. Mech. Des. 112, 369-376 (1990)

7. C.S. Liu, K. Zhang, R. Yang, Acta Mech. Sinica 21, 451-458 (2005)

8. C.S. Liu, K. Zhang, R. Yang, Mech. Mach. Theory 42, 183-197 (2007) 
9. Z.F. Bai, Y. Zhao, Int. J. Nonlin. Mech. 48, 15-36 (2013)

10. J. Ma, L. Qian, G. Chen, M. Li, Mech. Mach. Theory 94, 148-164 (2015)

11. J. Alves, N. Peixinho, M. Tavares da Silva, P. Flores, H.M. Lankarani, Mech. Mach. Theory 85, 172-188 (2015)

12. X.P. Wang, G. Liu, S.J. Ma, J. Mech. Sci. Technol. 30, 1537-1545 (2016)

13. D.F. Moore, Principles and Applications of Tribology. (Pergamon Press, Oxford, 1975)

14. R.S. Bradley, Philos. Mag. 13, 853-862 (1932)
15. B.V. Derjaguin, V.M. Muller, Y.P. Toporov, J. Colloid Interface Sci. 53, 314-326 (1975)

16. K.L. Johnson, K. Kendall, A.D. Roberts, Proc. R. Soc. London, Ser. A 324, 301-313 (1971)

17. D. Tabor, J. Colloid Interface Sci. 58, 2-13 (1977)

18. D. Maugis, J. Colloid Interface Sci. 150, 243-269 (1992)

19. J.M. Baney, C.-Y. Hui, J. Adhes. Sci. Technol. 11, 393-406 (1997)

20. T.X. Liu, G. Liu, Q. Xie, Q.J. Wang, ASME J. Tribol. 128, 40-48 (2006)

21. A.M. Guellil, J.B. Adams, J. Mater. Res. 7, 639-652 (1992) 